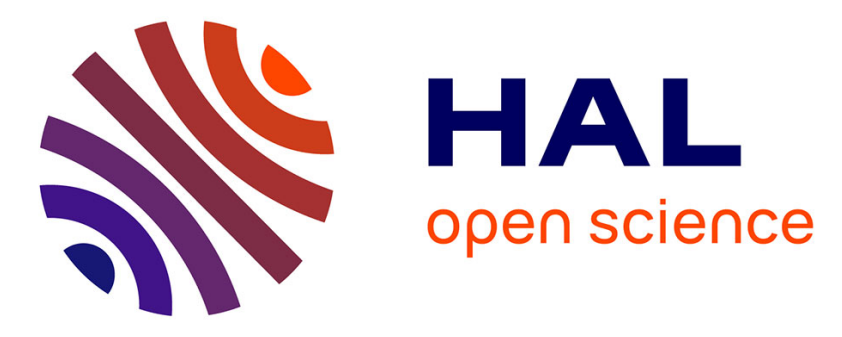

\title{
Second harmonic generation for contactless non-destructive characterization of silicon on insulator wafers
}

D. Damianos, L. Pirro, G. Soylu, I. Ionica, V. Nguyen, G. Vitrant, A. Kaminski, D. Blanc-Pélissier, L. Onestas, J. Changala, et al.

\section{To cite this version:}

D. Damianos, L. Pirro, G. Soylu, I. Ionica, V. Nguyen, et al.. Second harmonic generation for contactless non-destructive characterization of silicon on insulator wafers. Solid-State Electronics, 2016, 115, pp.237 - 243. 10.1016/j.sse.2015.08.006 . hal-01893414

\author{
HAL Id: hal-01893414 \\ https://hal.science/hal-01893414
}

Submitted on 11 Oct 2018

HAL is a multi-disciplinary open access archive for the deposit and dissemination of scientific research documents, whether they are published or not. The documents may come from teaching and research institutions in France or abroad, or from public or private research centers.
L'archive ouverte pluridisciplinaire HAL, est destinée au dépôt et à la diffusion de documents scientifiques de niveau recherche, publiés ou non, émanant des établissements d'enseignement et de recherche français ou étrangers, des laboratoires publics ou privés. 


\title{
SECOND HARMONIC GENERATION FOR CONTACTLESS NON-DESTRUCTIVE
}

\section{CHARACTERIZATION OF SILICON ON INSULATOR WAFERS}

\author{
D. Damianos ${ }^{*(1)}$, L. Pirro ${ }^{(1)}$, G. Soylu ${ }^{(1)}$, I. Ionica ${ }^{(1)}$, V. Nguyen ${ }^{(1)}$, G. Vitrant ${ }^{(1)}$, A. Kaminski ${ }^{(1)}$, D. Blanc- \\ Pelissier $^{(2)}$, L. Onestas ${ }^{(1)}$, J. Changala ${ }^{(3)}$, M. Kryger ${ }^{(3)}$, S. Cristoloveanu ${ }^{(1)}$
}

${ }^{*}$ Corresponding author: dimitrios.damianos@minatec.grenoble-inp.fr

(1) IMEP-LAHC, Minatec, Grenoble-INP, Univ. de Grenoble Alpes, CNRS-UMR 5130, 3 Parvis Louis Néel - CS 50257, F-38016 Grenoble, France

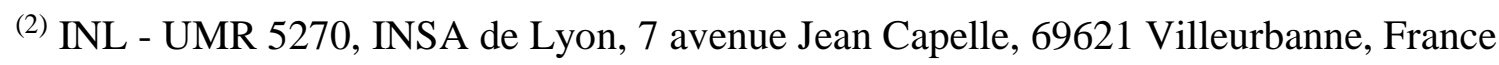

${ }^{(3)}$ FemtoMetrix, 1850 East Saint Andrew Place, Santa Ana, CA 92705, USA

Abstract - In this work we investigate a non-invasive, non-destructive characterization technique for monitoring the quality of film, oxide and interfaces in silicon-on-insulator (SOI) wafers. This technique is based on optical second harmonic generation (SHG). The principles of SHG and the experimental setup will be thoroughly described. The experimental parameters best suited for testing SOI wafers with SHG are identified. SOI geometry, as well as the passivation of the top surface, both have an impact on the observed SHG signal. The back-gate bias applied on the substrate is shown to modulate the SHG signal.

Keywords - Second Harmonic Generation, non-destructive characterization, SOI substrates, ultra-thin SOI, back-gate 


\section{Introduction}

There is a semiconductor industry need for in-line metrology tools capable of non-destructively characterizing SOI substrates with the throughput necessary for deployment in a high volume manufacturing environment. The chapter on the Front-End Process in ITRS 2013 [1] explains the quality requirements for silicon on insulator (SOI) wafers used for integrated circuit fabrication. These requirements relate not only to the quality of the interface between the silicon film (device layer) and buried oxide (BOX), but also the maximum acceptable values of the surface contamination, local thickness variations and other parameters.

In this context, optical second harmonic generation (SHG) [2] is an excellent non-destructive candidate due to its sensitivity to both material crystallographic quality and internal electrical field (induced by oxide charge, interface state density, surface contamination, etc). For example, Fiore et al. [3] probed silicon dopant type using time evolution of the SHG signal and showed that dopant concentration can be determined by the magnitude of the initial SHG signal at the time of measurement. Another study showed that in boron doped $\mathrm{Si} / \mathrm{SiO}_{2}$ systems, $\mathrm{SHG}$ could probe the boron-induced charge traps in the oxide via behavior versus time, depending on the oxide thickness [4]. For SOI, Alles et al. [5], [6] have demonstrated that the SHG technique can yield information about metal contamination in SOI wafers, which makes it valuable for microelectronics wafer quality assurance metrology. SHG is also capable of detecting the effects of irradiation on SOI wafers, as well as post-irradiation annealing effects, which is relevant for defense and aerospace applications [7].

In this paper we present our preliminary experimental results for SOI characterization using the novel optical SHG metrology system from FemtoMetrix [8]. We describe the operating principles of SHG in Section II and show the experimental set-up in Section III. The experimental parameters such as 
polarization angles, angle of incidence, and azimuthal angle were optimized for experiments on SOI in Section IV. The SHG results obtained on SOI wafers with various geometries and surface states are explained in Section V. Two interesting extensions of our experiment, one based on the back-gate effect and the other on the mapping possibilities, will be discussed at the end.

\section{SHG principle}

The optical second harmonic is a consequence of the second-order polarization generated in a material due to its interaction with high intensity light. Incident light with wavelength $\lambda$ (angular frequency $\omega$ ) induces polarization of different orders within that particular material. The second harmonic signal generated from this induced polarization has an intensity that is proportional to the polarization of order 2, $\overrightarrow{\mathrm{P}}^{(2)}$ (see Figure 1). For bulk materials (and in the absence of any electric field within the material), the second order polarization term is proportional to the square of the electrical field of the incident light $E(\omega)$ :

$$
\overrightarrow{\mathrm{P}}^{(2)}(2 \omega)=\vec{\chi}^{(2)} \cdot \overrightarrow{\mathrm{E}}(\omega) \cdot \overrightarrow{\mathrm{E}}(\omega)
$$

where $\chi^{(2)}$ is the second order susceptibility tensor of the material [9].

$$
\begin{aligned}
& \begin{array}{l}
\text { Incident monochromatic light, } \\
\lambda \text { (or } \omega \text { ) }
\end{array} \\
& \mathrm{I}^{\omega} \propto|\mathrm{E}(\omega)|^{2} \begin{array}{l}
\text { Generated second harmonic, } \\
\boldsymbol{\lambda} / 2 \text { (or } 2 \omega)
\end{array} \mathrm{I}^{2 \omega} \propto\left|\overrightarrow{\mathrm{P}}^{(2)}(2 \omega)\right|^{2}=\left|\vec{\chi}^{(2)}\right|^{2} \cdot|\mathrm{E}(\omega)|^{4} \\
& \text { Polarization of the material: } \\
& \overrightarrow{\mathrm{P}}=\overrightarrow{\mathrm{P}}^{(1)}+\overrightarrow{\mathrm{P}}^{(2)}+\overrightarrow{\mathrm{P}}^{(3)}+\ldots
\end{aligned}
$$

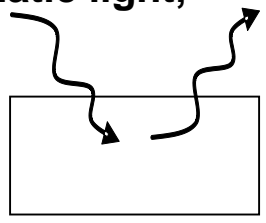

Figure 1: Principle of SHG. The incident laser of wavelength $\lambda$, angular frequency $\omega$ and intensity $I^{\omega}$ generally produces different order polarizations $P$ in the material. The intensity measured at $\lambda / 2$, notated $\mathrm{I}^{2 \omega}$ contains the second order polarization only. 
For centro-symmetric bulk materials such as $\mathrm{Si}$ or $\mathrm{SiO}_{2}, \chi^{(2)}$ is zero, so no second order polarization occurs. However, the broken symmetry near interface(s) between different materials leads to a non-zero interface $\chi^{(2)}$ contribution [10]. Furthermore, if a supplementary electric field (internal or applied) $E$ is present in the material, a second harmonic polarization related to the bulk third order nonlinear susceptibility will be proportional to $\chi^{(3)} E[10] . E$ is slowly varying as compared to optical frequencies and can thus be considered as quasi-static. This contribution - called EFISH (electric field induced second harmonic) - is generally modest because of the small values of $\chi^{(3)}$, unless the electric field is high. In the case of $\mathrm{Si}_{-} \mathrm{SiO}_{2}$ stacks, the electric field can be strong enough to reveal an EFISH contribution. Moreover, the electric field $E$ can evolve in time, either by varying the applied external field or due to internal field variation (e.g. charge trapping and de-trapping phenomena). In SOI all the interfaces (labeled "i") can contribute to the SHG signal, with both symmetry-breaking and EFISH terms. Consequently, the total intensity measured at the doubled frequency (for independent interface electric fields contributions) is given by [2]:

$$
\mathrm{I}^{2 \omega}(\mathrm{t}) \propto \sum_{\mathrm{i}}\left|\chi_{\mathrm{i}}^{(2)}+\chi_{\mathrm{i}}^{(3)} \cdot \mathrm{E}_{\mathrm{i}}(\mathrm{t})\right|^{2} \cdot\left(\mathrm{I}^{\omega}\right)^{2}
$$

Equation (2) provides a term accounting for the effects of the internal electric field, which is known to be related to interface state density and oxide charges.

\section{Measurement set-up}

The optical SHG wafer inspection system (Harmonic F1x ${ }^{\circledR}$ ) developed by FemtoMetrix was used for these measurements [8]. The experimental configuration is schematically shown in Figure 2. The pump laser delivers femtosecond pulses to the sample at $\lambda=780 \mathrm{~nm}$ wavelength. The minimum spot diameter on the sample is approximately $50 \mu \mathrm{m}$ for this tool but it increases with the angle of incidence 
of the incoming beam. The spatial resolution of the measurement is obviously related to the actual beam size on the wafer. Spot size can be adjusted on Harmonic F1 ${ }^{\circledR}$ systems based on test needs down to $\sim 5$ microns. A half wave plate (HWP in Figure 2a) is used to control the polarization orientation of the linearly polarized incident beam. A new beam (the second harmonic) is generated at the surface and interfaces of the wafer, at double frequency of the incident beam, i.e. at $\lambda=390 \mathrm{~nm}$ wavelength, and is detected by a photomultiplier tube, coupled with a photon counter (PMT in Figure 2a). A filter and a rotating polarizer allow the analysis of the polarization state of the SHG beam. The sample is placed on a metallic chuck which can accept wafers with diameters up to $300 \mathrm{~mm}$. A probe can be placed on the top surface of the wafer and a bias voltage can be applied between the probe and the chuck. Figure $2 b$ shows a picture taken inside the machine, with a 200mm-diameter wafer placed for measurement. More information about the tool can be found in [8].

(a)

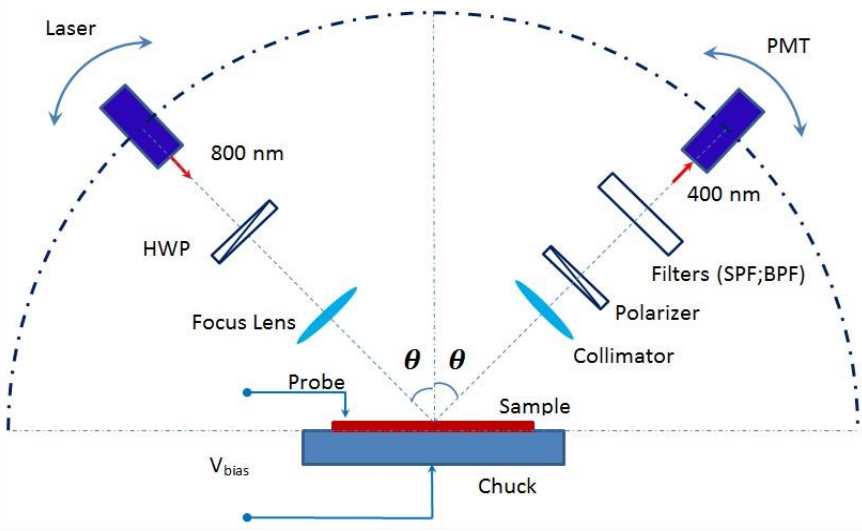

(b)

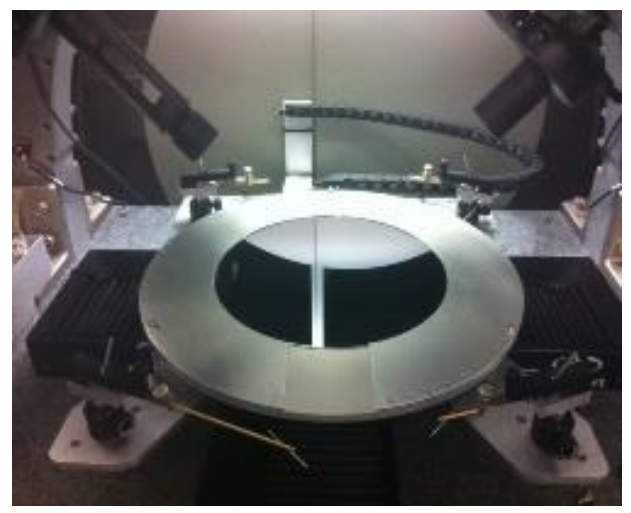

Figure 2: (a) Schematic representation of the measurement configuration for the SHG system from FemtoMetrix. (b) Picture of $200 \mathrm{~mm}$ diameter wafer placed on the chuck inside the machine.

A typical SHG intensity versus time graph is presented in Figure 3a. The particular SOI wafer under test had an $88 \mathrm{~nm}$ thick Si film, a $145 \mathrm{~nm}$ BOX, and a non-passivated surface (i.e., covered with native oxide). The silicon film on the top of the SOI was selectively etched, creating square SOI islands, 
separated by exposed BOX areas. This enables separate measurement of SHG on both SOI island (Si) and exposed BOX $\left(\mathrm{SiO}_{2}\right)$. As shown on Figure 3a, the signal coming from the BOX is about one order of magnitude lower than the one measured directly on the silicon film. This indicates that the SHG coming from the interface between the BOX and substrate does not dominate the signal when the measurement is performed on the silicon film. The second remark concerns the time evolution observed while probing the silicon film, which looks similar to a charging phenomenon. Figure $3 \mathrm{~b}$ schematically explains this behavior. Assuming a positively charged BOX layer (usual situation), a built-in electric field is present at the interface between the BOX and the Si film $\left(E_{\text {interface }}\right)$. When the laser is shined on the material, electron-hole pairs are generated and separated by the existing interface field. These free carriers interact with the oxide charges and the interface states, modifying the internal electric field. Depending on the relative signs of the contributions $\chi^{(2)}$ and $\chi^{(3)} E$ in equation 2 , the SHG signal can have either charging or discharging behavior. Consequently, the SHG intensity evolves with time. The initial SHG value is linked to the original internal electric field (dependent on interface states, oxide charges, etc.), while its time dependency is connected to the recombination dynamics of free carriers governed by the interfaces' quality.

The time of measurement of a single SHG curve depends on the information needed and material system under test. The initial electric field can be obtained within $100 \mathrm{~ms}$ (the shortest time for the photon counter gate opening is $10 \mathrm{~ms}$ ). On the other hand, in order to observe trap charging dynamics, the SHG signal needs to reach saturation value which can take longer depending on material systems, with $88 \mathrm{~nm} / 145 \mathrm{~nm}$ SOI results displayed below. (see Figs. 3a and 4).

Before going into any detailed physical explanation or modeling of the SHG signal from SOI, the experimental SHG parameters must be chosen in order to have the most relevant measurement 
configuration. The next section is dedicated to determining the optimum experimental SHG parameters (e.g., angles, polarizations etc.).

(a)

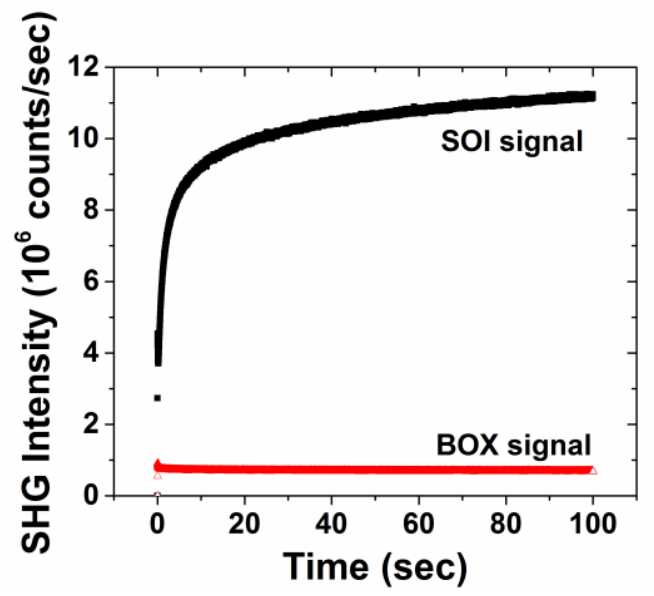

(b)

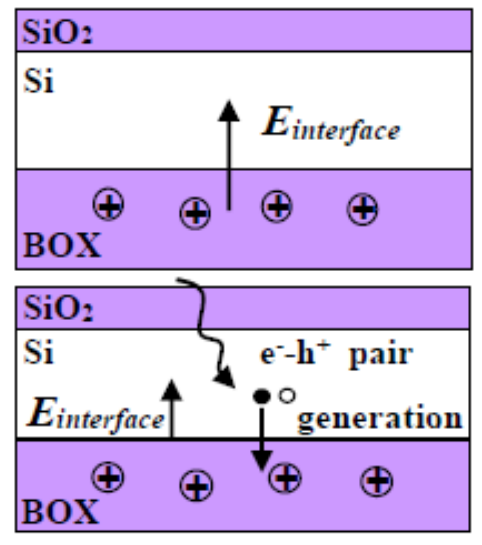

Figure 3: a) Typical SHG intensity vs. time measured on the BOX region and on the silicon film. The SOI under test had an 88nm film, a $145 \mathrm{~nm}$ BOX thicknesses, and a non-passivated top surface. The experimental configuration used was: $P$-in for incident beam polarization, $P-o u t$ for the SHG polarization; $45^{\circ}$ angle of incidence and $0^{\circ}$ azimuthal angle. b) Schematic representation of the phenomena appearing at the $\mathrm{Si} / \mathrm{SiO}_{2}$ interface before and after laser shining for a positively charged $\mathrm{BOX}$. The top $\mathrm{SiO}_{2}$ layer is either a native or a passivation oxide.

\section{Choice of SHG experimental parameters}

The aim in this section is to choose the most appropriate experimental parameters for the SHG measurement on SOI, in terms of input/output polarizations, angles of incidence and azimuthal angles.

\section{A. Input/Output polarization configurations}

Both the incident (input) and the SHG (output) polarizations can be modified with our measurement equipment. In the P-polarized state, the optical electric field oscillates parallel to the 
incidence plane, while in the S-polarized state oscillates perpendicular, i.e. parallel to the surface of the silicon wafer, regardless of the angle of incidence. Figure 4 shows, in semi-logarithmic scale, the different in/out polarization pairs from silicon film (Figure 4a) and exposed BOX (Figure 4b). The lowest signals are obtained in both cases with the S-out configuration, as theoretically expected [10]. For the P-out configurations, when S-in is used, the signal from the exposed BOX has the same order of magnitude as the one from the silicon film, which suggests that the substrate/BOX interface has a nonnegligible contribution in this experimental configuration. Since the aim of these measurements is to have a tool characterizing the interface between the top Si film and BOX, we prefer using P-in, P-out configuration, which gives the strongest signal from the film and for which the BOX contribution is one order of magnitude lower.

(a)

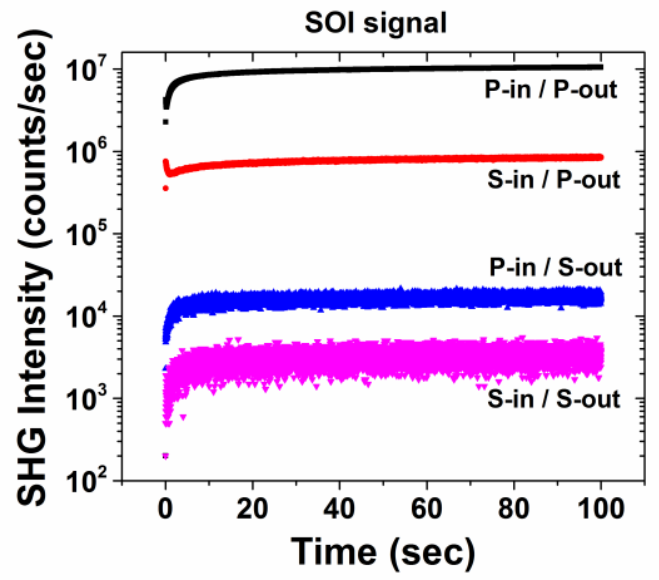

(b)

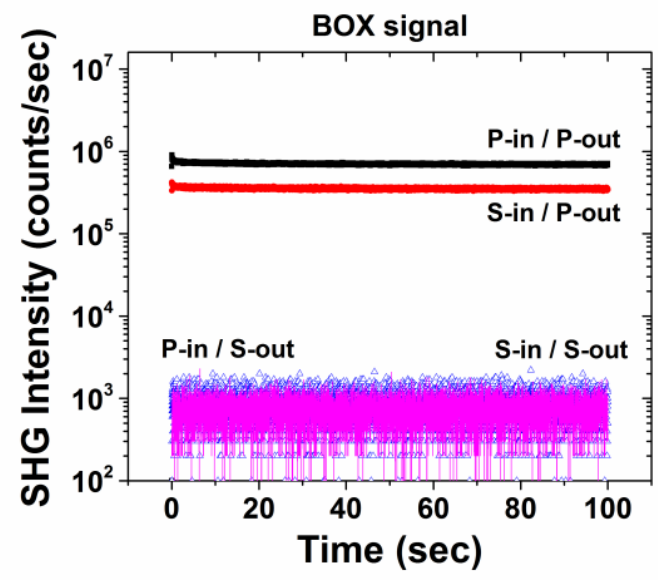

Figure 4: Polarization configurations of the incident and the SHG beams for SOI wafer with nonpassivated surface, $88 \mathrm{~nm}$ film and $145 \mathrm{~nm}$ BOX thicknesses. The angle of incidence was $45^{\circ}$. (a) Signal from silicon film region on the SOI island. (b) Signal from BOX region.

\section{B. Angle of Incidence}

Figure 5 shows the impact of the angle of incidence on the SHG intensity for the same SOI sample. The signal was collected on both the Si film and the exposed BOX for different angles of 
incidence. The maximum for the SOI signal appears at $45^{\circ}$. The BOX signal peak is shifted and, more importantly, it is at least one order of magnitude lower than for SOI. Subsequent experiments used a $45^{\circ}$ incidence angle in order to maximize signal from the silicon film.

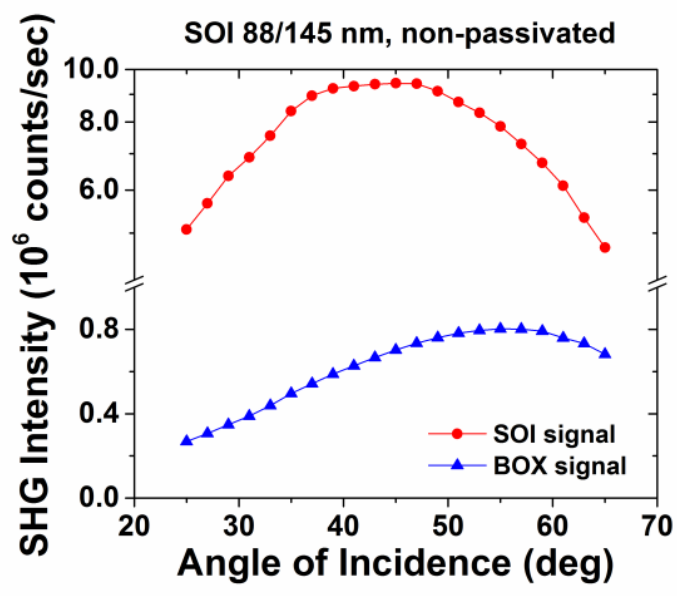

Figure 5: Dependence of the SHG signal on the angle of incidence. The incident and the reflected SHG beams were both P-polarized. Same SOI sample as in Figure 4.

\section{Azimuthal angle}

In a crystal, the SHG intensity depends on the angular orientation of the sample on the chuck, described by the azimuthal angle. For silicon (100) samples, it is known that the SHG shows a $90^{\circ}$ periodicity versus the azimuthal angle, which results from 4-fold symmetry [11]. Figure 6a shows our measurement results on SOI, using P-in/P-out polarizations and $45^{\circ}$ angle of incidence. 4 -fold symmetry is apparent, albeit the signal variation is less than $10 \%$. For validation we have performed measurements on control non-SOI wafers (thermally oxidized bulk Si) which show the same trend as the exposed BOX region (see Figure 6b). For pragmatic reasons related to the square shape of our SOI samples, we will use the $0^{\circ}$ azimuthal angle for next sections. 
(a)

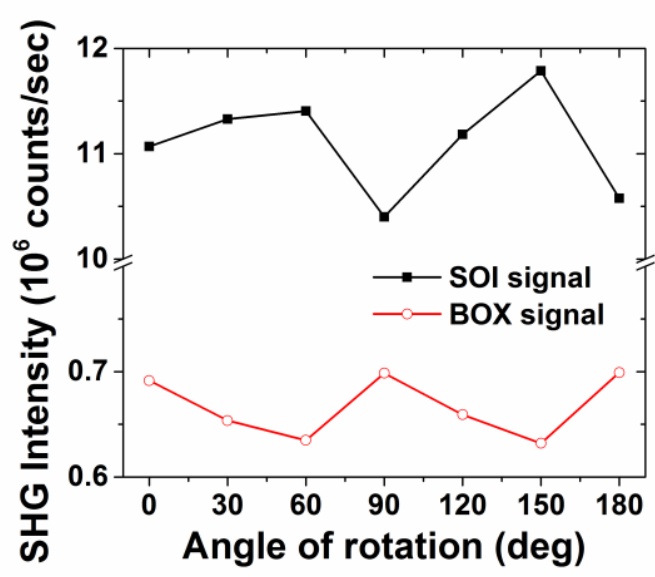

(b)

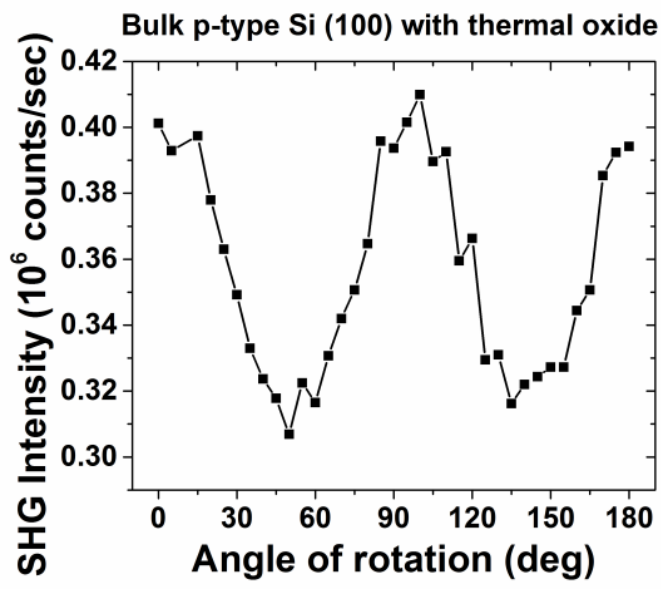

Figure 6: (a) SHG signals versus angle of rotation of the sample (azimuthal angle) in 88nm/145nm, non-passivated SOI. The probed areas were a Si island and an exposed BOX region. (b) SHG signal originating from control p-type bulk Si (100) with thermal oxide on top.

\section{SHG signal versus SOI parameters}

Based on the SHG results from $88 \mathrm{~nm}$ film and $145 \mathrm{~nm}$ BOX non-passivated SOI, we showed that the optimum SHG test parameters to characterize the interface between top Si film and BOX is obtained for the following configuration: P-in/P-out polarizations, $45^{\circ}$ angle of incidence and $0^{\circ}$ azimuthal angle. In this section we investigate the impact of different SOI parameters on the SHG signal. The areas under study were both the silicon film on the SOI island and the exposed BOX for each of the measured samples. Passivated and non-passivated samples were both tested in the study. The passivation process resulted in a 4nm thick dry thermal oxide which is known to reduce the top interface states' density [12].

\section{A. SHG from naked BOX region}

Figure 7 shows the SHG signal originating from a region between Si islands where the buried oxide is exposed. The differences between the samples are very small because the SHG signal coming from the $\mathrm{BOX}$ region is mainly due to the $\mathrm{SiO}_{2} / \mathrm{Si}(\mathrm{BOX} / \mathrm{Substrate})$ interface, which is rather identical 
for all samples. The passivation has small effect since it only modifies the top silicon film which has been etched off. The minor change in SHG is attributed to the supplementary high temperature process step associated with the passivation process that may have affected the BOX. The difference between the samples with $88 \mathrm{~nm}$ and $12 \mathrm{~nm}$ film thickness is marginal $(<5 \%)$, probably caused by modifications in the process steps during wafer manufacturing.

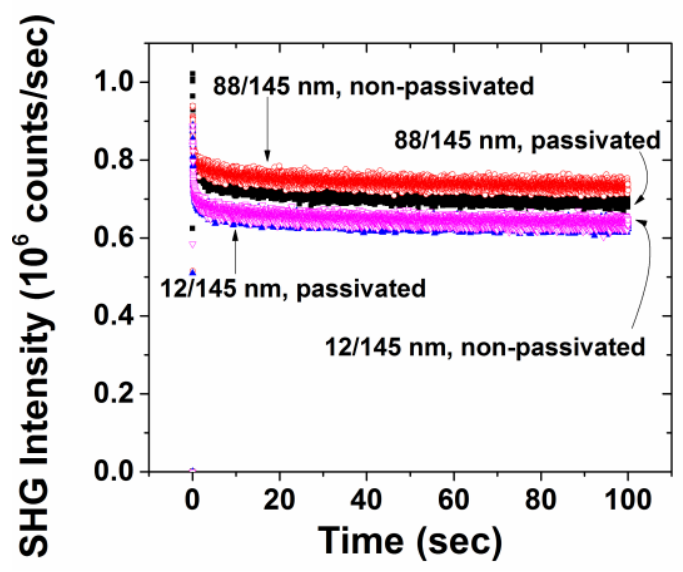

Figure 7: SHG signal originating from the $\mathrm{SiO}_{2} / \mathrm{Si}$ substrate interface. The probed area was an exposed BOX region between two subsequent SOI islands.

\section{B. BOX thickness impact}

Figure 8 depicts the time dependence of SHG signal measured on SOI samples with the same film thickness $(12 \mathrm{~nm})$ but varied BOX thicknesses $(145 \mathrm{~nm}, 25 \mathrm{~nm}, 20 \mathrm{~nm}$ and $15 \mathrm{~nm})$. Both the SOI island (Figure 8a) and the exposed BOX (Figure 8b) were tested. Note that the BOX is completely transparent to the incident and the SHG wavelengths; consequently, the SHG should not depend on the $\mathrm{SiO}_{2}$ thickness itself. However, fabrication steps for the different oxide thicknesses are slightly different, which can induce changes in the interface between the BOX and silicon substrate. The small differences in SHG coming from the BOX (Figure 8b) can likely be attributed to these changes. 
On the other hand, the signals measured from the SOI islands are more than one order of magnitude higher. The SHG intensity is similar in all samples with thin BOX layers (25, 20 and $15 \mathrm{~nm})$. However, we note a significant increase in SHG signal between the samples with the thin BOX and the sample with a thick BOX (145nm).

(a)

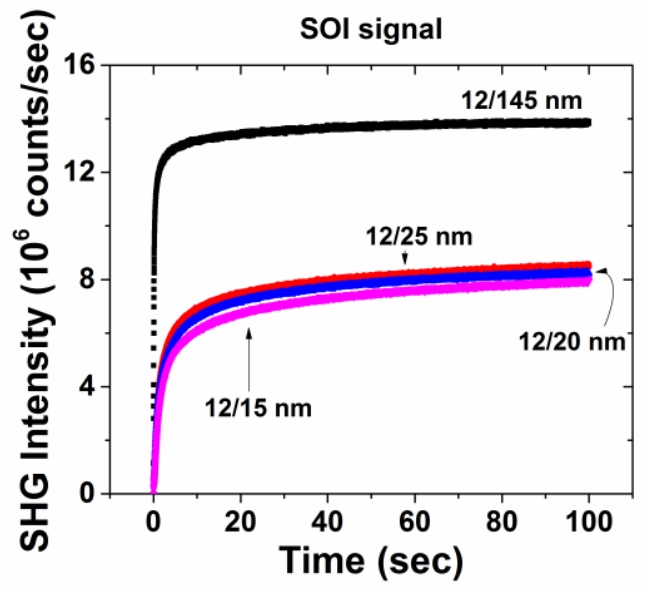

(b)

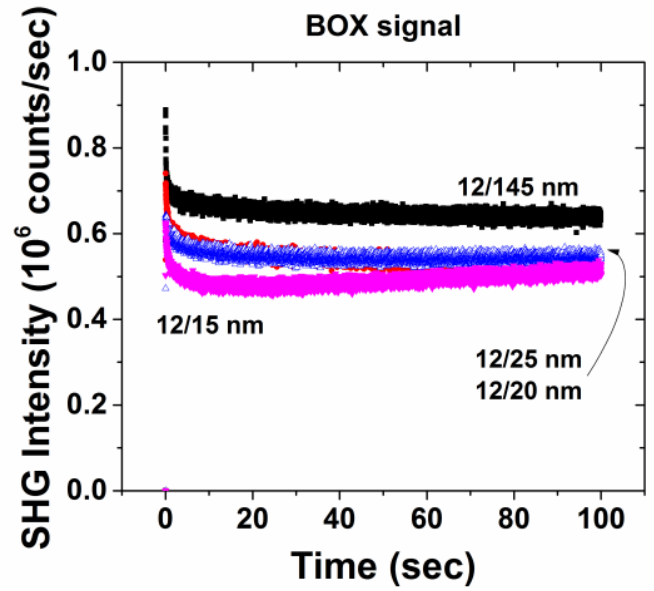

Figure 8: BOX thickness impact on SHG signal from non-passivated SOI. The top Si film thickness was $12 \mathrm{~nm}$ in all cases. The signals coming from the SOI film (a) and from the exposed BOX (b) are compared.

\section{Impact of Si film thickness and surface passivation}

In order to evaluate the impact of the Si film thickness and of surface passivation, we tested four SOI samples with $145 \mathrm{~nm}$ thick BOX layer. Two of the samples were passivated with $4 \mathrm{~nm}$ thermal oxide, while two of them were not passivated retaining a native surface oxide. Two different silicon thicknesses (88nm and 12nm) were compared.

A significant difference between passivated and non-passivated samples is evident for both $\mathrm{Si}$ film thicknesses (Figure 9). Passivated SOI generates less SHG signal, which is consistent with the reduction in the interface trap states during the passivation process [12]. This improved interface quality 
results in a smaller electric field in the SOI and consequently a lower SHG signal. There is also a slower time evolution for the passivated sample (clearly visible for the $88 \mathrm{~nm}$ film), presumably related to reduced carrier recombination rate in these samples.

In both passivated and non-passivated samples, the measured SHG signal is higher for the thinner silicon film, where the film-BOX interface is closer to the surface and contributes more to the SHG signal. We explain this result by the absorption of the exiting SHG signal by the silicon layer, which is related to the film thickness $\mathrm{t}_{\mathrm{si}}$. The absorption intensity is given by:

$$
I=I_{0} \cdot e^{-K \cdot t_{S i}}
$$

where $I_{0}$ is the initial intensity in the silicon film and $\mathrm{K}$ is the absorption coefficient of Si at 390nm SHG wavelength. The value of $\mathrm{K}$ used is $9.52 \times 10^{4} \mathrm{~cm}^{-1}$ [13]. $\mathrm{SiO}_{2}$ is transparent to $390 \mathrm{~nm}$ light and does not absorb any of the SHG signal. Therefore, the theoretical value for the ratio of the SHG intensities, due to absorption in Si films with different thicknesses, is:

$$
\frac{I_{88 / 145}}{I_{12 / 145}}=\frac{I_{0} \cdot e^{-K \cdot t_{S i 1}}}{I_{0} \cdot e^{-K \cdot t_{S i 2}}} \approx 0.47
$$

From Figure 9, we calculated the ratio of the SHG intensities at $\mathrm{t}=100 \mathrm{~s}$ and found a value of 0.81 for the non-passivated sample and 0.51 for the passivated sample. This indicates that, in the passivated SOI samples, the thickness dependence of the SHG intensity is well explained by the absorption of the $390 \mathrm{~nm}$ radiation in the silicon films. However, in the non-passivated samples, the measured ratio is a factor of two higher than the theoretical ratio, showing the increased importance of the electric field induced by surface charges. 
(a)

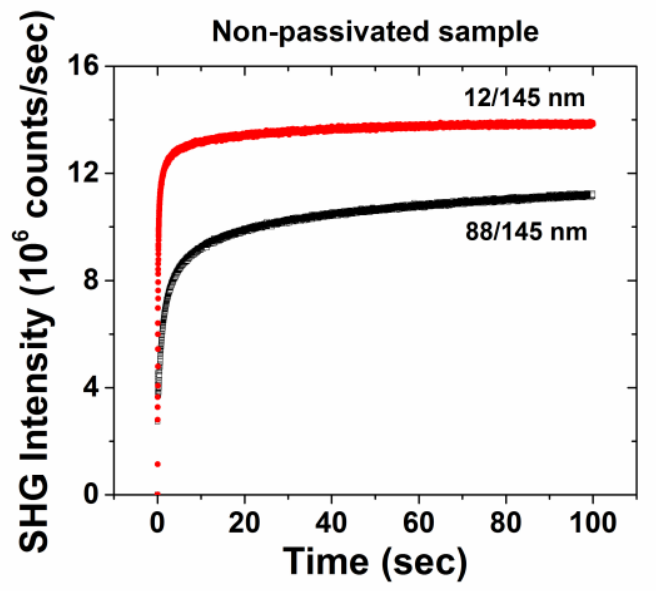

(b)

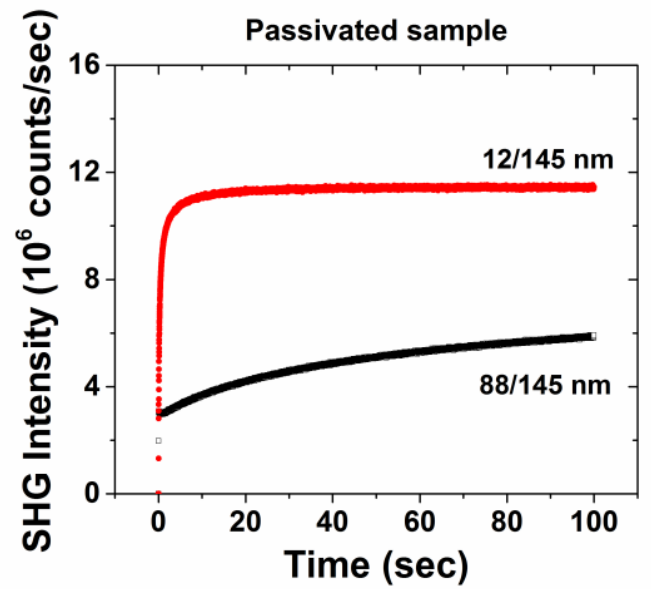

Figure 9: SHG signals measured on two non-passivated SOI samples (a) and two passivated SOI with thermal oxide on the top silicon film. All the samples had 145nm BOX thickness. Two Si film thicknesses were tested: $88 \mathrm{~nm}$ and $12 \mathrm{~nm}$.

\section{Possible SHG extensions}

\section{A. Back-gate bias effect on the SHG signal}

The conventional electrical tests for SOI wafers are based on the pseudo-MOSFET operation: mobile charges at the interface between the silicon film and BOX are induced by the voltage applied on the bulk silicon substrate used as a back gate [14]. Two probes, placed on the wafer surface, serve as source and drain contacts. The pseudo-MOSFET delivers accurate current-voltage characteristics from which the key material parameters are extracted: mobility of electrons and holes, threshold and flat-band voltages, etc. The drawback of this technique is that the wafer surface is locally damaged by the probes. Measuring an SHG signal modulated by the bias voltage applied on the substrate is a highly promising method to achieve a contactless pseudo-MOSFET. The relationship between the inversion/accumulation charge in the channel and the interface electric field is basic MOSFET knowledge [15], applicable to pseudo-MOSFETs. The idea is to use the SHG signal for monitoring the variation of the channel charge 
with gate bias. Preliminary comparisons between pseudo-MOSFET and SHG curves have been performed on irradiated SOI [7].

Figure 10a shows the SHG intensity versus time for different voltages $\mathrm{V}_{\text {bias }}$ applied to the substrate. A grounded probe is placed on the Si film; no gradient of the SHG signal with respect to the distance from the probe was observed. The initial intensity of the SHG signal as well as its time evolution are clearly modulated by $\mathrm{V}_{\text {bias. }}$ The full expression can be written by enriching Eq.(2):

$$
I^{2 \omega}(t) \propto\left|\chi^{(2)}+\chi^{(3)} \cdot\left(E_{\text {intrinsic }}+E_{\text {ext }}\left(V_{\text {bias }}\right)+E(t)\right)\right|^{2} \cdot\left(I^{\omega}\right)^{2}
$$

where $E_{\text {intrinsic }}$ is the pre-existing interface electric field, $E_{\text {ext }}$ is the applied electric field (due to $V_{\text {bias }}$ ) and $\mathrm{E}(\mathrm{t})$ is the time-dependent electric field due to charge redistribution. In order to emphasize the dependence of the SHG signal on the applied bias, Equation (3) can be rewritten as:

$$
\sqrt{\mathrm{I}^{2 \omega}(\mathrm{t})} \propto \chi_{\mathrm{i}}^{(2)}+\chi_{\mathrm{i}}^{(3)} \cdot\left(\mathrm{E}_{\text {intrinsic }}+\mathrm{E}_{\mathrm{ext}}\left(\mathrm{V}_{\text {bias }}\right)+\mathrm{E}(\mathrm{t})\right)
$$

In Figure 10b, we plotted the square root of SHG signal versus $\mathrm{V}_{\text {bias }}$ at given measuring times $\left(\mathrm{t}=0,30\right.$ and 300s, arrows in Figure 10a). The linear shape of the curves observed for high $\mathrm{V}_{\text {bias }}$ is well predicted by equation (6); indeed, in strong inversion, the external field is proportional to the inversion charge and therefore to the nominal voltage $\left(\mathrm{V}_{\text {bias }}-\mathrm{V}_{\mathrm{t}}\right)$. In the subthreshold region, at low $\mathrm{V}_{\text {bias, }}$, the curves become non-linear and require further modeling.

These results show that with pseudo-MOSFET based calibration, there is a clear path for SHG to give quantitative values of oxide charges and interface trap densities. This utility will be further discussed in future work. 
(a)

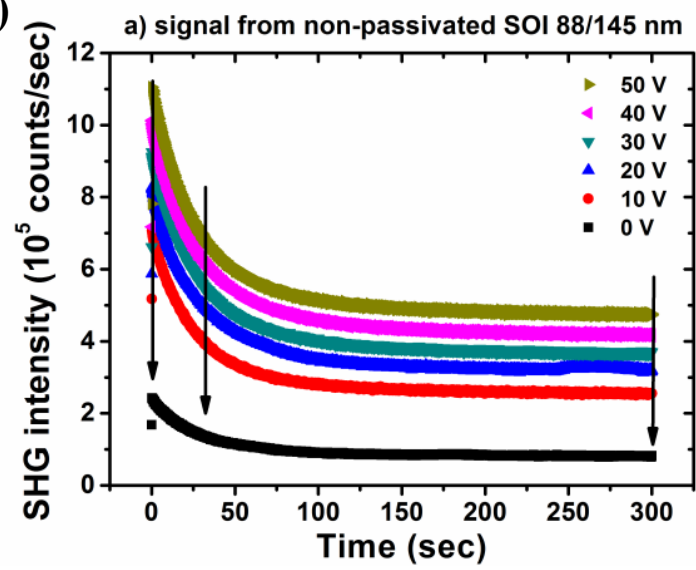

(b)

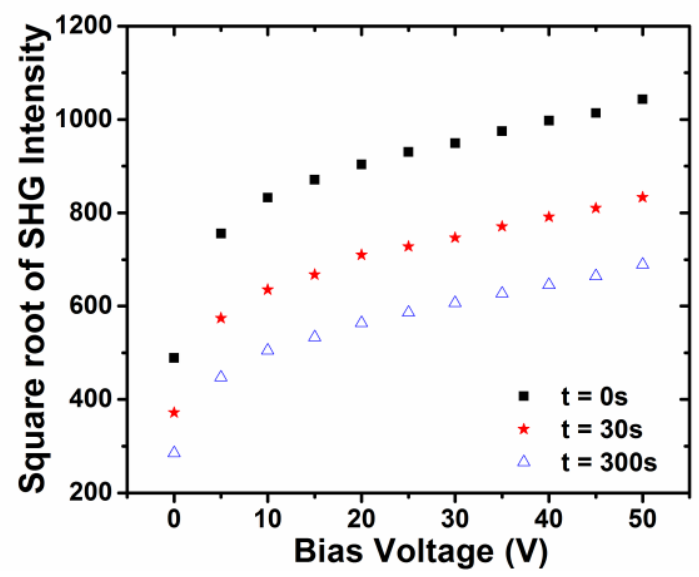

Figure 10: (a) SHG signal versus time for various biases applied on the substrate (used as a gate). The SOI wafer under testing had $88 \mathrm{~nm}$ thick Si film and $145 \mathrm{~nm}$ BOX. (b) $\sqrt{I^{2 \omega}}$ versus substrate voltage. The SHG intensities were extracted from the complete curves in Figure 9a at various times indicated by arrows.

\section{B. Mapping of SHG signal}

Figure 11 shows another interesting possibility offered by our SHG experimental tool: SHG mapping of SOI wafers. The observed square patterns correspond to the square islands of SOI, with each island having $4 \mathrm{~mm}$ on a side. These islands are typically used for pseudo-MOSFET characterization and the SHG image reveals differences between the islands which have been probe-damaged or not. This example shows the possibility to map in-situ and in real-time the SHG response across SOI wafers, nondestructively and without surface contact. Given the sensitivity of SHG to the quality of interfaces, these spatial maps are of utility for semiconductor process monitoring and quality control.

Note that the total time for wafer mapping depends on the spatial resolution desired. The measurement on each point can take anywhere from $100 \mathrm{~ms}$ up to several minutes, depending on the material system and information sought. 


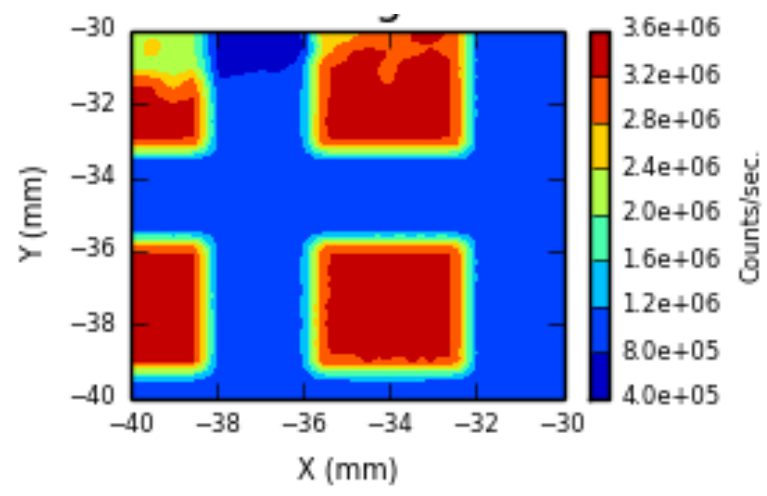

Figure 11: Mapping of SHG maximum intensity on a sample with $4 \times 4 \mathrm{~mm}^{2}$ SOI islands
surrounded by exposed BOX. The sample was a passivated $88 / 145 \mathrm{~nm}$ SOI wafer.

\section{Conclusions}

Second Harmonic Generation is demonstrated as a nondestructive method to monitor the quality of ultrathin SOI wafers. The experimental parameters leading to the strongest SHG signal from the film/BOX interface of SOI samples were P-in, P-out for the incident and SHG polarizations, $45^{\circ}$ angle of incidence and a rotation angle of the sample of $0^{\circ}$. The SHG method was tested on samples with different film and BOX thickness, as well as different top surfaces (passivated or not). First-order calculations showed that the absorption of the SHG by the silicon film can explain the results obtained in passivated samples with variable thickness: additional efforts are in progress to check whether the models developed in the literature for silicon apply to SOI.

Furthermore, the possibility to modulate the SHG signal by the voltage applied on the substrate was demonstrated. The high sensitivity of the SHG technique to indirectly probe the inversion charge can be used to calibrate the contactless SHG curves with electrical pseudo-MOSFET data. The results reported in this paper evidence the path for a non-contact measurement technique which is highly sensitive to interface states density and fully compatible with ultrathin SOI. 


\section{Acknowledgments}

Authors thank Drs. C. Mazuré, D. Delprat, I. Radu and O. Konokchuk from SOITEC for sample supply and inspiring discussions. The work was partly supported by Place2be and Compose3 European projects. The authors would like to thank the Region Rhône Alpes for financial support (ARC6 program) and the SHG team from Vanderbilt University for cooperation.

\section{References}

[1] http://www.itrs.net/Links/2013ITRS/Home2013.htm, 2013.

[2] B. Jun, Y. V. White, R. D. Schrimpf, D. M. Fleetwood, F. Brunier, N. Bresson, S. Cristoloveanu, and N. H. Tolk, "Characterization of multiple $\mathrm{Si} / \mathrm{SiO} 2$ interfaces in silicon-on-insulator materials via second-harmonic generation," Applied Physics Letters, vol. 85, pp. 3095-3097, 2004.

[3] J. L. Fiore, V. V. Fomenko, D. Bodlaki, and E. Borguet, "Second harmonic generation probing of dopant type and density at the Si/SiO2 interface," Applied Physics Letters, vol. 98, pp. 041905, 2011.

[4] H. Park, J. Qi, Y. Xu, K. Varga, S. M. Weiss, B. R. Rogers, G. Lupke, and N. Tolk, "Boron induced charge traps near the interface of $\mathrm{Si} / \mathrm{SiO} 2$ probed by second harmonic generation," Physica Status Solidi (b), vol. 247, pp. 1997-2001, 2010.

[5] M. L. Alles, R. Pasternak, X. Lu, N. H. Tolk, R. D. Schrimpf, D. M. Fleetwood, R. P. Dolan, and R. W. Standley, "Second Harmonic Generation for Noninvasive Metrology of Silicon-onInsulator Wafers," Semiconductor Manufacturing, IEEE Transactions on, vol. 20, pp. 107-113, 2007.

[6] M. L. Alles, D. M. Fleetwood, N. H. Tolk, R. D. Schrimpf, V. Koldyaev, M. Kryger, and J. Changala, "Rapid Non-Destructive Detection Of Sub-Surface Cu in Silicon-On-Insulator Wafers by Optical Second Harmonic Generation," (presented at 26th Annu. IEEE/SEMI Advanced Semiconductor Manufacturing Conference (ASMC), Saratoga Springs, New York, USA, vol. pp. 2015.

[7] B. Jun, R. D. Schrimpf, D. M. Fleetwood, Y. V. White, R. Pasternak, S. N. Rashkeev, F. Brunier, N. Bresson, M. Fouillat, S. Cristoloveanu, and N. H. Tolk, "Charge trapping in irradiated SOI wafers measured by second harmonic generation," Nuclear Science, IEEE Transactions on, vol. 51, pp. 3231-3237, 2004.

[8] http://www.femtometrix.com

[9] P. N. Butcher and D. Cotter, The Elements of Nonlinear Optics: Cambridge University Press, 1991.

[10] J. J. H. Gielis, P. M. Gevers, I. M. P. Aarts, M. C. M. van de Sanden, and W. M. M. Kessels, "Optical second-harmonic generation in thin film systems," Journal of Vacuum Science \&amp; Technology A, vol. 26, pp. 1519-1537, 2008.

[11] H. Tom, T. Heinz, and Y. Shen, "Second-harmonic reflection from silicon surfaces and its relation to structural symmetry," Physical review letters, vol. 51, 1983. 
[12] G. Hamaide, F. Allibert, H. Hovel, and S. Cristoloveanu, "Impact of free-surface passivation on silicon on insulator buried interface properties by pseudotransistor characterization," Journal of Applied Physics, vol. 101, pp. 114513, 2007.

[13] M. A. Green, "Self-consistent optical parameters of intrinsic silicon at 300K including temperature coefficients," Solar Energy Materials and Solar Cells, vol. 92, pp. 1305-1310, 2008.

[14] S. Cristoloveanu and S. Williams, "Point-contact pseudo-MOSFET for in-situ characterization of as-grown silicon-on-insulator wafers," IEEE Electron Device Letters, vol. 13, pp. 102-104, 1992.

[15] S. M. Sze, Physics of Semiconductor Devices. New York: John Wiley \& Sons, 1981. 